\title{
A prospective study on the effectiveness of intermittent ventilation of non- dependent lung in combating hypoxia during one lung ventilation
}

\author{
Sujith Alexander Kurian ${ }^{1, *}$, Jayakumar $\mathbf{P}^{2}$ \\ ${ }^{1}$ Assistant Professor, Dept. of Cardiac Anaesthesiology, ${ }^{2}$ Professor, Dept. of Cardiac Surgery, M.O.S.C. Medical College \& \\ Hospital, Kolenchery, Kerala, India
}

*Corresponding Author:

Email: lev3216@yahoo.co.in

Received: $26^{\text {th }}$ September, 2017

Accepted: $27^{\text {th }}$ November, 2017

\begin{abstract}
Introduction: One lung ventilation (OLV) is required for most of the lung surgeries and one of the common problem which we encounter during this surgical procedure is hypoxia. Our prospective study evaluates the effectiveness of a low tidal volume ventilation to the operated, non- dependent lung (NDL) in improving the oxygenation during OLV.

Materials and Methods: 40 ASA I, II and III patients posted for elective open thoracotomy was studied. After standard induction of anesthesia, lung separation was achieved with left sided DLT. ABG was taken on two lung ventilation (TLV) before thoracotomy and lung separation and that value was taken as base line. After thoracotomy the dependent lung alone (OLV) was ventilated for 15 mins and ABG was repeated. Then the NDL was ventilated with another ventilator with a low tidal volume and limited airway pressure and sample ABG were taken at 5 minutes and 20 minutes of NDL ventilation.

Results: ABG values of $\mathrm{PaO} 2$ suggested that thought the values decreased during OLV compared to base line values with TLV, $\mathrm{PaO} 2$ had a significant increase after instituting NDL ventilation.

Conclusion: NDL ventilation with low tidal volume and limited peak pressure is an effective method to avoid hypoxia and improve oxygenation during OLV requiring lung surgeries.
\end{abstract}

Keywords: One lung ventilation, Hypoxemia, Two lung ventilation.

\section{Introduction}

Human body has two lungs which are situated on the left and right in the thoracic cavity. Though they are morphologically two separate organs, they work as one functional unit. They function in tandem to provide adequate gas exchange and maintain normal levels of oxygen and carbon dioxide in blood. During lung surgeries it is desirable to isolate each lung which is taking part in the respiratory function, because in that way it helps the operating surgeon to have a better exposure of the intra thoracic structures and provide an optimal operating field. Various lung isolation techniques are being used which includes usage of bronchial blockers and double lumen tubes. One lung ventilation (OLV) refers to mechanical separation of the two lungs to allow ventilation of only one lung, while the other lung is compressed by the surgeon or allowed to passively deflate. At the time of OLV even though only one lung is ventilated both lungs are perfused and this leads to ventilation perfusion mismatch. This can lead to trans-pulmonary shunting, impairment of adequate oxygenation and finally leading to hypoxia. ${ }^{1-3}$

Hypoxia is a life threatening situation during lung surgery and various techniques have been used by anesthesiologists to prevent hypoxia during OLV. Using high inspired oxygen concentration $\left(\mathrm{FiO}_{2}\right)$ to dependent lung ventilation is the commonest method used to combat hypoxia during OLV. Some advocate the use of limited continues positive airway pressure
(CPAP) and intermittent re insufflations of the non dependent lung (NDL) in improving hypoxia during $\mathrm{OLV} ., 3$

\section{Aim and Objectives}

Our aim in this prospective clinical study is to evaluate the effectiveness of intermittent low tidal volume inflation of the NDL during one lung ventilation by comparing the variation in partial pressure of oxygen $\left(\mathrm{PaO}_{2}\right)$ at stipulated time interval during OLV. We also study if this technique of NDL ventilation during OLV interferes with the operating surgeon's easiness in conducting surgery.

\section{Materials and Methods}

The study was presented before the Ethics Committee of our institution and approval was obtained. Written informed consent was obtained from all the patients enrolled in the study. Forty patients were selected for the study which was determined by calculating the statistical power. The optimal sample size of forty patients participating in the study provided a power of $80 \%$ to find a one tailed difference of $30 \mathrm{mmHg}$ in partial pressure of oxygen $\left(\mathrm{PaO}_{2}\right)$ at the time of OLV considering an $\alpha$ error of $5 \%$ based on an expected standard deviation of $25 \mathrm{mmHg}$. Our patient inclusion criteria were all patients posted for elective thoracotomy with duration more than sixty minutes and between the age group of 18 years to 70 years who comes under ASA I, II and III. We excluded patients of 
age less than 18 years and more than 70 years. All patients with any cardiac or other severe co morbidities were also excluded from the study. Thoracoscopy cases were also excluded from the study.

All patients were subjected to the same anesthesia induction and maintenance protocol. Patients were premedicated with Diazepam 10mg oral the night before surgery and on the day of surgery. In the operating room, intra venous cannula (18G or $16 \mathrm{G})$ and radial artery (20G) lines were aseptically secured. All patients received pre-oxygenation with $100 \%$ oxygen while lines were secured. All patients were induced with Midazolam $0.1 \mathrm{mg} / \mathrm{kg}$, Fentanyl 5-10mg/kg, Propofol $1 \mathrm{mg} / \mathrm{kg}$ and Sevoflurane at 0.5 to 1 minimum alveolar concentration (MAC). After confirming smooth bag and mask ventilation, Pancuronium $0.01 \mathrm{mg} / \mathrm{kg}$ was used as the muscle relaxant and all patients underwent tracheal intubation with left sided DLT (Rusch). The correct placement of the double lumen tube was confirmed first by auscultation method. A Fiberoptic Bronchoscope (FOB) was used to reconfirm the position of the DLT in the supine position first and later on in the lateral position.

All patients received thoracic epidural catheters and thoracic epidural anesthesia was initiated. A continues infusion of Bupivacaine $0.125 \%$ and Fentanyl $2 \mathrm{mcg} / \mathrm{ml}$ was loaded in a $50 \mathrm{ml}$ syringe and infused though a syringe pump at $5 \mathrm{ml} / \mathrm{hr}$ as thorasic epidural anesthesia. Isoflurane and boluses of fentanyl was used to maintain comfortable hemodynamics. All patients after tracheal intubation were ventilated with inspired oxygen fraction $(\mathrm{FiO} 2)$ of 0.6 with a GE Health Care Avance Ventilator. We selected the PRVC mode with a tidal volume of $8-10 \mathrm{ml} / \mathrm{kg}$, respiratory rate of $12-14 /$ minute, I:E ratio of $1: 2$, and a PEEP of $5 \mathrm{~cm} \mathrm{H}_{2} \mathrm{O}$. This mode of ventilation was continued till lung isolation in lateral position. An ABG was taken after 10 minutes of two lung ventilation (TLV) in the above mentioned settings and those values were taken as the base values in our study.

All patients were placed in later decubitus position, either left or right for the surgery. Dependent lung ventilation or OLV was initiated just before puncturing the pleura after thoracotomy. Complete collapse of the non dependent lung was confirmed visually by the operating team. The lumen of the DLT corresponding to the nondependent lung was kept open to room air to facilitate complete lung collapse during thoracotomy. After 15 minutes of one lung ventilation, an ABG sample was collected and that values were recorded. Then we connected the nondependent lung to another ventilator (Macquet Servo i). This NDL was ventilated in PRVC mode with tidal volume of $75 \mathrm{ml}$, respiratory rate of 8 per minute, I:E ratio of $1: 6, \mathrm{FiO} 2$ of 0.75 and with no PEEP. ABG samples were repeated at 5 minutes and 20 minutes after starting NDL ventilation with the above mentioned settings. ABG was analyzed at $5 \mathrm{~min}$ and $20 \mathrm{~min}$ after starting the nondependent lung ventilation. If any patient during the time of OLV started desaturating $(\mathrm{SpO} 2<90 \%)$, then $\mathrm{FiO} 2$ was increased to 1 and ventilated till recovery of oxygen saturation. The operating surgeon was to make an assessment about ease of doing surgery on a partially ventilating lung, which was also recorded on a verbal scale of 1 to 10, where a score of 1 implies that the surgical dissection is impossible and a score of 10 would mean that the lung collapse is well adequate for the smooth conduct of surgery. Any patient with profound hypoxia $(\mathrm{SpO} 2<80 \%)$ was abandoned from the study and rescue protocol was instituted. Rescue protocol for profound hypoxia was to reconfirm position of DLT with FOB followed by through suctioning of the DLT lumens and both lung ventilation with $\mathrm{FiO}_{2}$ of 1, after informing the surgeon.

\section{Statistical methods}

Continuous variables (blood gas values drawn at different times) were summarized as mean \pm standard deviation and were compared across the groups using Student's paired $t$-test. A $P<0.05$ was considered statistically significant. SPSS for Windows, Version 16.0 (SPSS Inc., Chicago, IL, USA) was used for the analysis.

\section{Results}

Forty patients were consecutively enrolled for this study. Of theses 40 patients, 28 were males and 12 were females. The patient details and surgical procedures are presented in table 1 and table 2 . None of the patients enrolled in the study had any significant cardiac diseases or other co morbidities.

\section{Table 1: Demographic data}

\begin{tabular}{|l|c|}
\hline Patients & 40 \\
\hline Males & 28 \\
\hline Females & 12 \\
\hline Age $(\mathrm{yrs})$ & $52.6 \pm 14.8$ \\
\hline Height $(\mathrm{cms})$ & $162.3 \pm 10.4$ \\
\hline Weight $(\mathrm{kg})$ & $61.8 \pm 12.3$ \\
\hline
\end{tabular}

Left sided double lumen tube was used in all patients. Right thoracotomy was done in 25 cases and left thoracotomy in 15 patients. Out of forty patients, twenty eight patients recorded a drop in oxygen saturation below $95 \%$ and four patients recorded drop in saturation below $90 \%$ when subjected to OLV. All these patients improved saturation within 3 minutes of starting NDL ventilation.

Table 2: Surgical Data

\begin{tabular}{|l|c|}
\hline Right thoracotomy & 25 patients \\
\hline Left thoracotomy & 15 patients \\
\hline Left sided DLT & 40 patients \\
\hline Decortication & 11 patients \\
\hline Wedge resection & 3 \\
\hline Aspergilloma & 3 \\
\hline
\end{tabular}




\begin{tabular}{|l|c|}
\hline Lobectomy & 7 \\
\hline Lung Biopsy & 3 \\
\hline Segment resection & 5 \\
\hline Lung trauma & 3 \\
\hline Bullectomy & 5 \\
\hline $\begin{array}{l}\text { Duration of surgery } \\
\text { (mins) }\end{array}$ & $104 \pm 38.2$ \\
\hline $\begin{array}{l}\text { Duration of OLV } \\
\text { (mins) }\end{array}$ & $72 \pm 26.3$ \\
\hline
\end{tabular}

From Table 3 we can analyze the arterial blood gas results which were recorded during our study. It shows that there was a decrease in $\mathrm{PaO}_{2}$ when the patient was put on OLV from TLV with values of $201 \pm 45.8$ during ABG 1 TLV coming down to $104.3 \pm 32.5$ during ABG 2 OLV with $P<0.001$.

Table 3: Arterial blood gas results (expressed in mean \& standard deviation)

\begin{tabular}{|l|c|c|c|c|}
\hline \multicolumn{1}{|c|}{ Parameter } & ABG 1 TLV & ABG 2 OLV & ABG 3 OLV & ABG 4 OLV \\
\hline $\mathrm{pH}$ & $7.34 \pm 0.05$ & $7.34 \pm 0.03$ & $7.31 \pm 0.02$ & $7.33 \pm 0.05$ \\
\hline $\mathrm{PaO}_{2}(\mathrm{mmHg})$ & $201 \pm 45.8$ & $104.3 \pm 32.5$ & $154.1 \pm 46.3$ & $174.7 \pm 49.8$ \\
\hline $\mathrm{PaCO}_{2}(\mathrm{mmHg})$ & $44.2 \pm 7.42$ & $46.3 \pm 7.38$ & $41.7 \pm 7.6$ & $40.2 \pm 7.1$ \\
\hline $\mathrm{Hb}(\mathrm{g} / \mathrm{dl})$ & $13.6 \pm 2.3$ & $13.2 \pm 1.7$ & $12.7 \pm 1.4$ & $12.2 \pm 1.5$ \\
\hline
\end{tabular}

TLV: two lung ventilation, OLV: one lung ventilation, $A B G$ 1: after putting DLT, $A B G$ 2: after clamping NDL, $\mathrm{ABG}$ 3: 5 minutes after NDL Ventilation, ABG 4: 20 minutes after NDL Ventilation.

The ABG which was taken 5 minutes and after 20 minutes after starting NDL ventilation showed a remarkable improvement in $\mathrm{PaO} 2$ to $154.1 \pm 46.3$ and $174.7 \pm 49.8$ respectively compared with the ABG 2 value of $\mathrm{PaO} 2$ which was only $104.3 \pm 32.5$ with $P<$ 0.001. A graphical representation of the partial pressure of oxygen recorded in our study is shown below:

\section{Graph 1:}

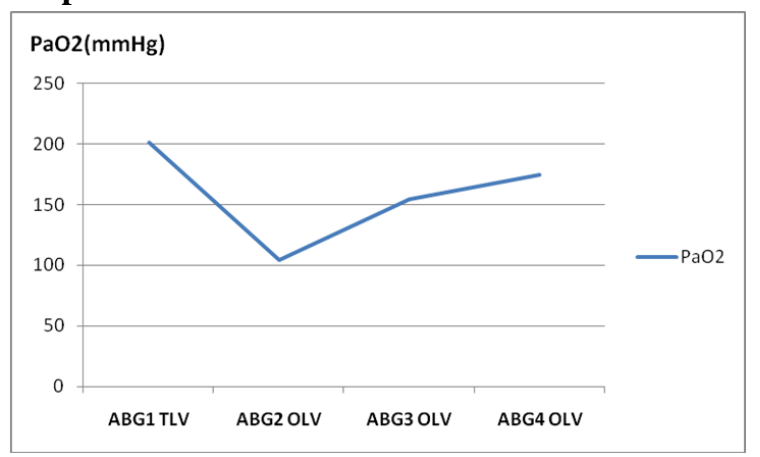

There were no significant changes in $\mathrm{pH}$ and $\mathrm{PaCO}_{2}$ in the ABG results during TLV, OLV or during NDL. The operating surgeon gave a score of 10 in 36 cases and 8 in 4 cases which implies that the mild lung excursion during NDL ventilation did not affect the ease of doing the surgery.

\section{Discussion}

One lung ventilation (OLV) is a common term used in thoracic anesthesia to provide ventilation only to a desired lung and to isolate the other lung. It is commonly used for three main purposes: to improve surgical access, lung protection and rarely for intensive care ventilation. There are 3 main ways by which we can provide OLV in anesthesiology. They are by using bronchial blockers, double lumen tubes (DLT) and by using a small sized single lumen endotrachel tube which is inserted beyond the carina into one of the bronchus. The most commonly used is a DLT tube and preferably a left sided DLT.

Hypoxia is one of the commonest problems every anesthesiologist face during one lung ventilation. ${ }^{1}$ Chances for hypoxia are higher in surgeries on the right lung, supine position and patients with coexistent lung diseases. Several factors play a role in the development of hypoxia in OLV. They are reduced oxygen reserve in the dependent lung, ventilation perfusion mismatch in the ventilated and collapsed lung and factors which reduce the effect of hypoxic vascular constriction. Oxygen reserve will be reduced in the dependent lung due to compression effect over it by the contents of the mediastinum and by the pressure exerted by the abdominal contents over the diaphragm during relaxation. Though the NDL lung is not ventilated at all, some perfusion will be maintained and this will lead to ventilation perfusion mismatch. ${ }^{1,2}$ Factors which reduce the effect of hypoxic pulmonary vasoconstriction includes rising pulmonary artery pressures, supine position, inadequate collapse of NDL lung due to adhesions and use of vasodilator drugs during surgery.

Several ventilator strategies have been studied to prevent hypoxia during OLV. This includes alveolar recruitment maneuvers, continues positive airway pressure (CPAP), positive end expiratory pressure (PEEP), fiber optic oxygen insufflations, selective lobar insufflations and non dependent lung ventilation. ${ }^{3,5,7}$ In alveolar recruitment maneuvers, atelectasis of lung is reversed by means of a brief, controlled increase in the airway pressure with expansion of the lungs. CPAP has been in use for many years to combat the problem of hypoxia during OLV. Studies have also shown that the use of CPAP has been beneficial in reducing reperfusion injury on the collapsed lung. Applying PEEP to the dependent lung has shown to be beneficial in certain case studies as it restore the FRC to almost normal value. 
Our study showed that intermittent ventilation of the nondependent lung with small tidal volume was successful in combating the problem of oxygen desaturation during OLV without affecting the surgical field. During OLV, even though the NDL is collapsed, perfusion is continued and this leads to shunt and hypoxemia. By using the reasoning stated by Russel, ${ }^{3}$ we could calculate the presumed oxygen deficit in our patients. Our patients had a hemoglobin level of average $12 \mathrm{gm} / \mathrm{dl}$. Since our patients were all cardiac stable, we assumed a cardiac output of $5 \mathrm{~L} / \mathrm{min}$ and $100 \%$ oxygen saturation. Then the Oxygen delivery will be about $800 \mathrm{ml} / \mathrm{min}$. Even if the patients deasaturated during OLV to around $80 \%$, then the oxygen deficit should be around $150 \mathrm{ml} / \mathrm{min}$. The ventilator settings used in our study with a tidal volume of $75 \mathrm{ml}$, ventilator rate of $8 / \mathrm{min}$, and $\mathrm{FiO} 2$ of 0.75 would supply oxygen in excess of $400 \mathrm{ml} / \mathrm{min}$ which is more than adequate to counteract the hypoxemia occurring during OLV.

Oxygen delivery may be calculated as follows: $\mathrm{DO} 2=$ $\mathrm{CO} \times \mathrm{CaO} 2 \times 10$.

where $\mathrm{CO}$ is cardiac output and $\mathrm{CaO}_{2}$ is the arterial oxygen content.

$\mathrm{CaO} 2=(\mathrm{Hb} \times 1.39 \times \mathrm{SaO} 2)+(\mathrm{PaO} 2 \times 0.003)$.

Where $\mathrm{SaO}_{2}$ is the oxygen saturation and $\mathrm{PaO}_{2}$ arterial oxygen tension.

Pressure regulated volume control (PRVC) mode was used for ventilating the NDL because it provides the set target tidal volume at the lowest peak pressure. ${ }^{4}$ The peak pressure never exceeded more than $10 \mathrm{cmH}_{2} \mathrm{O}$ with the set tidal volume in none of our cases. NDL ventilation with the set parameters were continued throughout the surgery period and the lung excursion remained at a level that was not detectable by the surgeon and did not affect his surgical dissections. Minimum air leaks which were present during the surgery were compensated by the Ventilator.

Ishikawa in his study showed that hypoxia during OLV will not continually deteriorate and in most of the cases it improves with time. ${ }^{5}$ However, keeping patient's safety in mind, corrective measures to treat hypoxia should be taken by the anesthesiologist. The NDL need not be ventilated throughout the surgery fearing hypoxia. None of our patients reported fall in saturation to $<95 \%$ even after discontinuing NDL ventilation after 20 mins. The reason is that following repeated alveolar collapse, the vascular resistance of lung increases leading to decrease in the shunt fraction and thus maintaining oxygen saturation for longer periods of time. Pirlo et al in his study has clearly stated that hypoxic pulmonary vasoconstriction is potentiated by repeated lung collapse. ${ }^{6}$ Studies have shown that a low CPAP of $2-5 \mathrm{cmH}_{2} \mathrm{O}$ is beneficial for improving hypoxia during OLV but is effective only when the lung is inflated to $30 \mathrm{~cm} \mathrm{H}_{2} \mathrm{O}$ for 15 seconds prior to it. ${ }^{7}$ This ventilator strategy may interfere with the surgical field and may not be successful in all the lung surgery patients. Selective segmental insufflations of oxygen into the non-operated lobe of the NDL using a fiber optic bronchoscope was studied by $\mathrm{Ku}$ et al, but the effectiveness is still under trial. ${ }^{8}$ Slimani et al has stated that regular inflation of oxygen even without a CPAP has shown to improve oxygenation during OLV. ${ }^{9}$

To evaluate which all patients will improve oxygenation from PEEP to the dependent lung during OLV will depend on the individual's lower inflection point on the static compliance curve. Only those patients who have low end expiratory pressure to begin with and application of PEEP elevating the end expiratory pressure to the lower inflection point will only get relieved of hypoxia when applying PEEP to dependent lung ventilation. ${ }^{10}$

\section{Limitations}

First of all, this study is done in a relatively small group of patients and thus needs to be confirmed in a larger clinical trial. Another limitation is that we did not include thoracoscopy surgery in our study group.

\section{Conclusion}

A brief period of low tidal volume positive pressure ventilation to the non dependent lung is a simple and cost effective technique to minimize hypoxia during OLV. Its added advantage lies in the fact that it least interferes with the surgical field. This technique provides an effective way to combat hypoxia during OLV.

\section{Financial support and sponsorship: Nil}

Conflicts of Interest: There are no conflicts of interest

\section{References}

1. Karzai W, Schwarzkopf K. Hypoxemia during one-lung ventilation: Prediction, prevention, and treatment. Anesthesiology 2009;110:1402-11.

2. Yasuuji M, Kusunoki S, Hamada H, Kawamoto M. Intermittent re-inflation is safe to maintain oxygenation without alteration of extra vascular lung water during one-lung ventilation. J Clin Anesth 2014;26:177-83.

3. Russell WJ. Intermittent positive airway pressure to manage hypoxia during one-lung anaesthesia. Anaesth Intensive Care 2009;37:432-4.

4. Guldager H, Nielsen SL, Carl P, Soerensen MB. A comparison of volume control and pressure-regulated volume control ventilation in acute respiratory failure. Crit Care 1997;1:75-77.

5. Ishikawa S. Oxygenation may improve with time during one-lung ventilation. Anesth Analg 1999;89:258-9.

6. Pirlo AF, Benumof JL, Trousdale FR. Potentiation of lobar hypoxic pulmonary vasoconstriction by intermittent hypoxia in dogs. Anesthesiology 1981;55:226-30.

7. Hogue CW Jr. Effectiveness of low levels of nonventilated lung continuous positive airway pressure in improving arterial oxygenation during one-lung ventilation. Anesth Analg 1994;79:364-7.

8. Ku CM, Slinger P, Waddell TK. A novel method of treating hypoxemia during one-lung ventilation for thoracoscopic surgery. J Cardiothorac Vasc Anesth 2009;23:850-2. 
9. Slimani J, Russell WJ, Jurisevic C. An evaluation of the relative efficacy of an open airway, an oxygen reservoir and continuous positive airway pressure $5 \mathrm{~cm} \mathrm{H}_{2} \mathrm{O}$ on the non-ventilated lung. Anaesth Intensive Care 2004;32:75660.

10. Slinger PD, Kruger M, McRae K, Winton T. Relation of the static compliance curve and positive end-expiratory pressure to oxygenation during one-lung ventilation. Anesthesiology 2001;95:1096-102. 